\section{Kenya comprehensive school health policy: lessons from a pilot program}

\author{
Job Wasonga, Betty Ojeny, Gordon \\ Oluoch, Ben Okech \\ CARE International in Kenya, Kisumu, \\ Kenya
}

Abstract

The study assessed the implementation of Kenya comprehensive school health pilot intervention program. This pilot program has informed the Kenya Comprehensive School Health Policy which is a critical document in the achievement of Millennium Development Goals relating to child health, gender equality, universal education and environmental sustainability. The study was based on focus group discussions, field observations and in-depth interviews with government officers who implemented the pilot program. The findings were categorized into implementation process, what is working well, what is not working well and lessons learned. During the course of the study, it was noted that involvement of all stakeholders enhances program ownership and sustainability but if they are not well coordinated or where supportive supervision and monitoring is not carried out, then some components of the comprehensive school health program may not be sustainable. We learnt that comprehensive school health program increases students' enrolment, attendance and retention, factors that are very important in a country's human resources development. The study has shown that although the formulation of a policy may be participatory and bottom-top, the implementation requires allocation of enough resources and coordination to bridge the gap between policy formulation and implementation.

\section{Introduction}

The Kenya comprehensive school health policy is based on the realization that education as a social determinant is affected by health and vice versa. It follows on the global spirit of health in all policies movements which articulates that public health community should not only be concerned with policies that shape health, but also with those that shape the primary determinants of health such as social, cultural, economical and environmental. ${ }^{1,2}$ The policy came as realization that good health is essential for the success of the implementation of any educational program in order to achieve desirable quality learning out- comes. ${ }^{3}$ The holistic approach to improvement of health in schools is aimed at achieving access, retention, inclusion, equity and completion of learners so as to achieve sustainable development as part of a wider strategy to achievement of vision 2030, a policy road map to make Kenya a middle income state by the year 2030 through implementation of projects touching on the economic, social and political aspects of the country.

As a commitment to achieving Millennium Development and Education for All Goals, the government of Kenya piloted comprehensive school health program in two model districts in the coastal region of the country targeting thirty primary schools. This pilot program, which was funded through the support of Japan International Cooperation Agency (JICA) later on, gave birth to comprehensive school health policy and was adopted after a wide consultation and contribution from different stakeholders both in government and other development agencies. The aim of this policy is to ensure that health of children including those of adolescent is taken care of in school and in the community. ${ }^{5}$

The policy covers a wide range of issues and tries to address different components of health which have been clustered into eight thematic areas, namely: i) values and life skills; ii) gender issues; iii) child rights, protection and responsibilities; iv) water, sanitation and hygiene; v) nutrition; vi) disease prevention and control; vii) special needs, disabilities and rehabilitation; viii) school infrastructure and environmental safety.

The policy is envisioned to meet greater proportion of health and psychosocial needs of children in the school and community. ${ }^{3}$ To achieve this, the government of Kenya has come up with National School Health Guidelines and Kenya Comprehensive School Health Handbook to operationalize the policy by ensuring that school age children, teachers, support staff and community access quality and equitable services for improved health.,5 The guideline and handbook is also to offer framework for school administrators, teachers, health workers and community leaders to guide ideas and organize activities so as to identify health issues in their schools and community in order to mitigate against factors which may hamper health and learning.

\section{The policy background}

The Kenya Comprehensive School Health Policy was developed after a pilot and practical experiences in Kilifi and Msambweni districts in the coastal region. The policy is to enable government to utilize available resources in an effective and efficient manner towards child health. It also provides coordination mechanism to enhance the roles of various min-
Correspondence: Job Wasonga,

CARE International in Kenya, Box 88 Kisumu, Kenya.

Tel. +254723686375 .

E-mail: wasonga@ksm.care.or.ke

Key words: Kenya, school health, policy, comprehensive school health policy.

Acknowledgements: the authors would like to thank the Medical Officers of Health, District Public Health Officers and the School Health Coordinators of Kilifi and Msambweni Districts, Ministry of Public Health and Sanitation, Kenya. The learning and sharing visit was made possible through SWASH+ program which is funded by Bill and Melinda Gates Foundation.

Received for publication: 24 December 2012.

Revision received: 19 December 2013.

Accepted for publication: 4 February 2014

This work is licensed under a Creative Commons Attribution NonCommercial 3.0 License (CC BYNC 3.0).

(C) Copyright J. Wasonga et al., 2014

Licensee PAGEPress, Italy

Journal of Public Health in Africa 2014; 5:313

doi:10.4081/jphia.2014.313

istries, institutions and stakeholders in tackling the inequality that exists in health provision especially preventive and promotive aspect of health. ${ }^{6}$

Kilifi is one of the districts in Kilifi County with a total population of 1.1 million. Females form $52 \%$ of the entire population while the school going children including under five form $47 \% .^{7}$ There are 160 primary and $23 \mathrm{sec}$ ondary schools with pupil enrolment of 154 , 848 in primary and 61,112 in secondary schools respectively, making it one of the counties with lowest transition from primary to secondary level and completion rates despite the teacher to pupil ratio of 1:36 in primary schools and 1:30 in secondary schools $68.5 \%$ of the residents of this county are poor according to Kenya poverty line ${ }^{7}$ and engage in subsis tence farming as one of the major economic activities. There is a high prevalence of malaria, gastro enteritis and diarrheal diseases among the communities residing in this area. There are different ethnic communities with diverse cultural practices some of which impact negatively on education and health. 0 n the other hand, Msambweni district is situated in the southern part of the coastal region in Kwale County. Like the sister Kilifi County, Kwale is one of the counties with high poverty rates, estimated at $74.6 \%$ according to Kenya poverty line and over $40 \%$ of the population live in absolute poverty. ${ }^{7}$ The population stands at 649,931 with females making $51 \%$ of the total population. The school going age children 
including the under fives represent $47 \%$ of the population. There are 349 primary and $41 \mathrm{sec}$ ondary schools respectively with an enrolment of 157,454 and 15,461 students in primary and secondary schools respectively. The pupil's teacher's ratio stands at 1:57 students in primary and 1:35 in secondary schools. ${ }^{7}$ Like Kilifi County, there is a low transition and completion rates due to many factors. The major economic activity of this region is small-scale agriculture alongside mining and fishing. It is under such circumstance that the Kenyan government piloted the comprehensive school health policy to inform the rest of the country and bring school health to scale.

The policy has been developed based on the National Health Sector Strategic Plan (NHSSP II) and in recognition that disease, disability and ill health are major impediments to development of any nation. ${ }^{8}$ Improved health in schools allows for better physical and cognitive development of children and therefore making them more productive members of the society. ${ }^{9}$ The policy defines comprehensive school health program and envisions that all partners or players in the education sector will embrace the approach in order to address not only the education and health needs of children and learners, but their families, teachers and communities. The policy compliments other policies that exist touching on health and education and as such advocates for the design and development of programs in the school system which addresses the needs of learners comprehensively and holistically to achieve sustained national development.

\section{Materials and Methods}

Sustaining and Scaling School Water, Sanitation and Hygiene plus Community Impact (SWASH+) program conducted a peer review exchange visit as part of its broader learning and policy advocacy approach to understand the background under which this policy was piloted, implemented and later on scaled all over the country. The SWASH+ program is a partnership between CARE International, Emory University's Center for Global Safe Water and Great Lakes University of Kisumu, and the Government of Kenya, with funding from Bill and Melinda Gates Foundation and Global Water Challenge. This is an applied research program whose aim is to develop, test and bring to scale innovative approaches to school water, sanitation and hygiene (WASH) approaches in primary schools and communities in western Kenya. One of the priority areas identified for achieving effective and sustained school WASH service in Kenya is by improving the sharing of knowledge among parents and pupils to teach- ers and school administrators to government, community and other development-sector participants. ${ }^{10}$

In April 2012, the researchers from SWASH+ program conducted a peer review exchange visit to understand and evaluate the comprehensive school health policy and program in the piloted districts. Prior to the visit, the researchers agreed on the specific areas and objectives that would guide the gathering of information from the schools that were to be visited for the purposes of learning. The objectives were:

- Share success stories from implementation of the pilot program and find out the major activities which have contributed to successful implementation of the pilot program. - Learn the challenges faced and how they were and have been tackled or addressed.

- Know how the pilot program has been able to manage and strengthen collaboration, networking and partnership in order to achieve success.

- Know how school WASH has been integrated into the broader school health policy and program.

- Understand the strategies for scaling up of the school health policy in Kenya.

The researchers engaged the district public health officers to identify schools in extremes, doing well and not doing well and also some schools that were in the middle. Seven schools were visited, 5 in Kilifi and 2 in Msambweni districts respectively. Through in- depth interview and group discussions with head teachers, health patrons, district public health officers, public health officers, school health coordinators and monitoring and evaluation officers, an insight into the comprehensive school health program pilot was obtained. A transect walk through the school was conducted and the conditions of the infrastructure including sanitary facilities in the schools visited were observed.

Although there are 20 primary schools in Kilifi and 10 primary schools in Msambweni districts where the comprehensive school health program was piloted, only 7 were visited due to constraint in time and other logistics. After every field visit from each of the districts, feedback was given to the district health management team on our findings for corrective action and to share our experiences.

\section{Results}

Based on consensus and guided by the objectives, which were earlier agreed upon, we were able to categorize our findings into implementation process, what is working well, what is not working well and lessons learned.

\section{Implementation process}

We found that before the implementation of the pilot program, the government conducted a survey to define baseline indicators in all the primary schools in the pilot districts. Targeting was also done for the selection of the 20 and 10 schools in Kilifi and Msambweni respectively. This baseline survey also covered health indicators.

The results from the baseline survey were shared at the zonal level where all the major stakeholders and the schools where it was done were present. Each school selected for the pilot program implementation developed a plan of action based on the weakness noted during the baseline survey analysis based on the 8 thematic areas of the policy.

The action plan developed was then shared with other teachers and school management committee (SMC) members who never attended stakeholders meeting at the school level. The outcome of these sharing meetings was that the schools identified activities to be done based on consensus and their ability to solve them. It also gave them the opportunity to identify other partners around their schools who can assist them tackle the problems identified in during the baseline survey. The baseline survey also acted as a benchmark for monitoring and evaluation of the pilot programmed in the implementing schools.

The implementation process also included implementation of the schools actions plans which involved assigning of responsibility to teachers, sensitization of pupils and teachers, involvement of SMC and other stake players on resource mobilization and allocation and lastly, frequent monitoring and supportive supervision by JICA and Ministry of Public Health and Sanitation officers (public health officers).

\section{What is working well}

We noted that through the involvement of schools in the baseline survey, schools were able to identify all their weak areas, share with people concerned with the management of the school and come up with their own solutions. This we observed, are captured in the schools action plans which were acting as a guide and a checklist to the implementation of the program to the school administrators. The involvement of everybody also ensured ownership of the program.

The training and participation of health teachers as well as other school teachers in the review and implementation of the schools action plans ensured school level ownership and sustainability.

Monitoring of the latrine cleanliness and other hygiene issues by the pupils themselves or prefects and orientating them on the policy thematic areas facilitated the uptake of the concept.

Involvement and participation of the SMCs 
is working well in terms of maintenance of the school facility such as infrastructures and resources allocation. Most of the schools visited had piped water and therefore, were able to maintain hygiene issues such as cleaning of latrines twice a day, washing of hands at critical times such as after visiting the latrines since all the latrines of the schools visited had hand washing station next or adjacent to the latrines. Hand washing station design has helped to sustain hand washing where they are constructed of concrete and situated next to or inside the latrines.

Linkage and partnership with all most all stakeholders in education sector in the pilot areas were involved in the initial stages, especially during the pilot intervention program. Schools had knowledge on where to get assistance, for example concerning nutrition, agricultural or farm and health issues but later on became weak.

\section{What is not working well}

We found that the coordination and networking between the government ministries, especially the ministry of public health and sanitation and that of education were weak. This was seen in the lack of participation of the divisional and zonal levels officers in the post pilot implementation where the government officers of these ministries were not working together.

Monitoring support from the District, Division and zonal levels ceased after the implementation of the pilot period leading to negative impact on the sustainability of the program.

There are no references materials for teachers and health patrons in the schools to enhance health education and hygiene promotion. This poses a challenge in ensuring sustainability of hygiene education and practices. We also noted the absence and lack of standardization of latrine construction and cleaning supplies. Children were observed cleaning latrines using twigs and makuti brooms without any detergents or disinfectants. This exposes the children to the risks of contracting infections as it was also observed that majority of the children population lacked shoes and were walking bare foot. Lack of shoes exposes children to worm infestation especially when visiting dirty latrines where their skin is most likely to come into contact with worms.

All the schools visited lacked anal cleansing materials for the learners and it was observed that many of the latrine walls were dirty with fecal content. It was also observed that some learners use the corners of the latrine doors to wipe themselves. The lack of anal cleansing materials for the children exposes them to hand contamination and subsequent infections as a result of dirty or faucal contaminated hands.
Considering that this policy advocates for a comprehensive approach to school health touching on the eight thematic areas, we found that not all (the thematic areas of the school health program) are functional. And in most of the schools visited, access to nutritional services such as school feeding has collapsed due to widespread poverty while the WASH component seems to be given prominence and support thereby overshadowing other areas. Other areas such as life skills, environmental and structural safety, although integrated into the school's curriculum and daily activities, they are not given priority that they deserve.

Some of the cultural practices such as children not being allowed to share latrines with adults and the belief system on ghosts hampers the delivery of comprehensive school health policy. We observed that due to cultural practices and belief system, the use of latrine and observation of hygienic behaviors such as cleaning and accessing of latrines are hampered in some of the schools visited. In one group discussion, it was participants noted that ...pupils report of seeing majini in the school latrines preparing meals, and in some cases seeing very tall people in the latrine. This hinders the usage of such facilities.

\section{Lessons learnt}

We have learnt that comprehensive school health program appears to increase enrollment, retention and attendance of pupils as was observed by the researchers. Participation of various stakeholders is key in implementation and sustainability of the comprehensive school health program. This we also learnt can be achieved through participation of multinational companies who have embraced the idea of corporate social responsibility as was observed in schools where tourists' hotels are nearby. Corporate and community responsibilities improves learning environment drastically, especially in infrastructure provision thereby increasing student enrollment, retention and attendance.

Provision of latrines without consideration of anal cleansing materials can have some negative unintended outcomes, as it will aid the spread of infections from one child to the other. Transferring of trained teachers and other people during implementation period weakens the program sustainability.

Although, in majority of the schools visited, we observed the presence of enough latrine facilities as well as water storage provision such as water tanks, there is lack of standardization of the latrine structures. Majority of the schools visited did not have recommended latrine structures due to lack of information and schools management not seeing any sense in taking their structural plans for approval by competent government authorities. We also observed that in these schools, there is a deliberate effort to provide piped water and electricity for the learners. Provision of water to schools or access to water is important to maintenance of hygiene and overall good health of students and community.

We also learnt that in few of the schools visited, pupils are encouraged to carry soap for hand washing from home to school. This is positive health behavior but the schools administration reported that they did not know whether all pupils' practices hand washing with soap and were not capable of monitoring this behavior.

The comprehensive school health handbook gives the governance, institutional and implementation structure for the operationalization of the policy. We observed that the operationalization of the institutional framework for implementation of the program has not been done and some of the committees such as school health committee have never been formed. The relationship between different government ministries, supportive supervision and coordination are not yet clear to stakeholders especially schools. This may hamper implementation, scale up and sustainability of comprehensive school health programm.

\section{Discussion}

Studies have shown that the process of policy implementation is usually divorced from the development of the policy itself and lack of proper planning may pose a great risk ${ }^{11}$ and hinder its implementation. The implementation process of the Kenya comprehensive school health policy is an approach that has hitherto been used in most development of policies in the country, bottom-top and which is the right direction in policy formulation. The pilot project provided a good platform to involve the citizens in policy formulation and learning in order to improve on the implementation. And as observed by Brynard, ${ }^{12}$ policy implementation is not merely an administrative choice which once legislated and the institutions mandated with administrative authority identified will just happen, but rather a process that encourages action by public and private individuals or groups towards achieving the objectives of the policy. According to this study, the implementation of this policy has been marred by factors such as inadequate funding, non-operationalization of the coordinating structure leading to poor networking and collaboration as well as lack of referencing materials for implementers.

Lack of enough funding to implement public health care interventions is not something new in Kenya, just like most countries in sub Sahara Africa, where implementation of poli- 
cies especially those that address promotive and preventative health such as the comprehensive school health policy, Kenya government has not provided enough resources to effectively implement this policy. Studies show that there is a large gap between policy formulation and implementation in Kenya ${ }^{13}$ and this affects development. Without enough resources the coordinating mechanism of the implementation suffers since prioritization of activities that can cause the desired change are not done or may not be followed. The Kenya comprehensive school health policy has eight thematic areas of which if not well coordinated, as is the case, then some areas of priority are likely to lag behind and therefore the achievement of the desired change may not be attained. For example, it is evident from the study that water, sanitation and hygiene is given more prominence than other thematic areas. This is due to funding from other partners who are advocating for universal and sustainable sanitation and drinking water both in schools and communities. While this is good and enhances provision of services to schools and communities coming from the national level, there is the lack of fostering partnership and collaboration which ensures sustainability at the local level. Networking and collaboration at the local level ensures ownership and sustainability thereby achieving policy objective, in this case, improved health of children in school and in the community to sustain human resources and development.

Networking and collaboration requires a strong coordinating mechanism that ensures effective monitoring and support supervision is carried out, best practices captured and challenges addressed to ensure implementation of the policy is on track. Lack of information and referencing materials for the implementers can be tackled if the coordinating mechanism is strong and ensures timely monitoring and supervision. Lack of referencing materials on the thematic areas such as water, sanitation and hygiene concerning latrine and water facility construction standards, hygiene education and promotion materials, agriculture and nutrition, life skills and disease control may hamper the implementation of the policy and thereby scuttle the achievement of its objec- tive. It has been observed in this study that majority of the schools visited which took part in the pilot program lack referencing materials to guide implementers in the implementation process of this policy yet it is being rolled down to the whole country. This challenge may therefore slow down its uptake and kill the spirit of its implementation.

\section{Conclusions}

The results of this study show that there is a lot to be done to bridge the gap between policy formulation and implementation of the Kenya comprehensive school health policy although the process of formulating it has been participatory. The quest of Kenyan government to transform the way policies are formulated and implemented to improve the health of her citizen is something which has to be lauded. Although the study has shown that there are issues which have to be tackled to achieve the objective of the policy, with planning and operationalization of the coordinating structures, the implementation of this policy will ensure that the health of Kenyan children and their communities is improved.

\section{References}

1. Siddiqi A, Kawachi I, Berkman L, et al. Education determines a nation's health, but what determines educational outcomes? A cross-national comparative analysis. J Public Health Policy 2012;33:115.

2. Puska P. Health in all policies. Eur J Public Health 2007;17:328.

3. Government of Kenya. National School Health Policy. Ministry of Public Health and Sanitation and Ministry of Education. Nairobi: Republic of Kenya; 2009.

4. Government of Kenya. Kenya vision 2030: the popular version. Ministry of Planning and National Development. Nairobi: Republic of Kenya; 2007.

5. Government of Kenya. National School
Health Guidelines. Ministry of Public Health and Sanitation and Ministry of Education. Nairobi: Republic of Kenya; 2009.

6. Government of Kenya. Kenya Comprehensive School Health Implementation Handbook. Ministry of Public Health and Sanitation and Ministry of Education. Nairobi: Republic of Kenya; 2009.

7. Government of Kenya. District poverty data KIHBS, 2005/06; 2005. Available from: http://www.opendata.go.ke Accessed: 11/04/2012.

8. Ministry of Health. National Health Sector Strategic Plan II (2005-2010). Nairobi: Republic of Kenya; 2005.

9. Albonico M, Allen H, Chitsulo L, et al. Controlling soil-transmitted helminthiasis in pre-school-age children through preventive chemotherapy. PLoS Negl Trop Dis 2008;2:e126.

10. CARE International, Emory University, Great Lakes University of Kisumu and Government of Kenya. Sustaining and Scaling School Water, sanitation and Hygiene Plus Community Impact Programme. Translating research into national-scale change: a case study from Kenya of WASH in schools. Nairobi: CARE International, Emory University, Great Lakes University of Kisumu and Government of Kenya; 2011.

11. Oyaya C0, Rifkin SB. Health sector reforms in Kenya: an examination of district level planning. Health Policy 2003;64: 113-27.

12. Brynard PA. Policy implementation: lessons for service delivery. African Association for Public Administration and Management, 27th AAPAM Annual Roundtable Conference, 5th-9th December 2005, Livingstone, Zambia.

13. Glenngard AH, Maina TM. Reversing the trend of weak policy implementation in the Kenyan health sector? - a study of budget allocation and spending of health resources versus set priorities. Health Res Policy Systems 2007;5:3. 